\title{
A comparison of staggered and non-staggered grid Navier-Stokes solutions for the 8:1 cavity natural convection flow
}

\author{
S. Armfield* R. Street ${ }^{\dagger}$
}

(Received 17 November 2004, revised 14 July 2005)

\begin{abstract}
The Navier-Stokes equations may be discretised using finite-volume schemes on non-staggered or on staggered grids. The staggered grid is known to prevent pressure oscillations that may occur on the nonstaggered grid; however, this is at the expense of increased code complexity. Non-staggered grid schemes that employ iterative time integration are known to require the use of some form of explicit correction in the construction of the Poisson pressure correction equation. We investigate a fractional-step pressure-correction non-staggered scheme and compare it to a similar fractional-step staggered grid scheme for bifurcated natural convection flow in an 8:1 cavity.
\end{abstract}

*School of Aerospace, Mechanical \& Mechatronic Engineering, Sydney University, Sydney, Australia. mailto:armfield@aeromech.usyd.edu.au

${ }^{\dagger}$ Environmental Fluid Mechanics Laboratory, Stanford University, Stanford, California 94305-4020, USA

See http://anziamj.austms.org.au/V46/CTAC2004/Armf for this article, (C) Austral. Mathematical Soc. 2005. Published September 12, 2005. ISSN 1446-8735 


\section{Contents}

1 Introduction

C919

2 Method

C924

2.1 Accuracy . . . . . . . . . . . . . . . C926

2.1.1 Divergence error . . . . . . . . . . . . . C C926

2.1.2 Velocity and pressure error . . . . . . . . . . . C928

2.1.3 Boundary condition error . . . . . . . . . . . . . C929

3 Results and discussion

C930

4 Conclusions

C932

References

C933

\section{Introduction}

Natural convection flow in a cavity with height/width aspect ratio of eight was used as a benchmark flow for unsteady Navier-Stokes solvers at a special session of the First MIT Meeting on Computational Fluid and Solid Mechanics [1]. The two-dimensional cavity has heating and cooling applied to the two vertical walls while the floor and ceiling are insulated. This configuration exhibits unsteady flow at Rayleigh number $\mathrm{Ra}=3.4 \times 10^{5}$ and Prandtl number $\operatorname{Pr}=0.71$, where the Rayleigh number $\mathrm{Ra}=g \beta \Delta T W^{3} /(\nu \alpha)$, with $g$ gravity, $\beta$ the coefficient of thermal expansion, $\Delta T$ the temperature difference between the heated and cooled walls, $W$ the cavity width, $\nu$ the kinematic viscosity and $\alpha$ the thermal diffusivity. The Prandtl number $\operatorname{Pr}=\nu / \alpha$. The basic configuration is shown in Figure 1.

The flow exhibits waves travelling up the hot wall and down the cold wall, circulating continuously around the cavity, and is only marginally supercriti- 


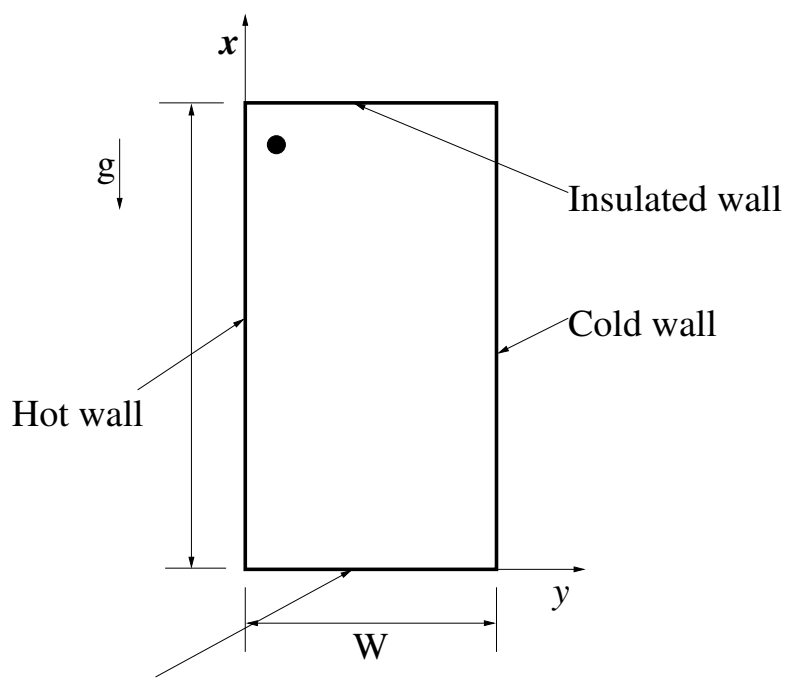

Insulated wall

FIGURE 1: Geometry and boundary conditions.

cal. Figure 2 shows instantaneous temperature and streamfunction contours for the fully developed flow, showing the basic structure with narrow natural convection boundary layers formed adjacent to the vertical walls, a stable stratification in the interior, and a cavity scale circulation. The unsteady nature of the flow is clearly seen in the temperature time series shown in Figure 3, which exhibits a sinusoidal oscillation. The wave-like structure of the flow is seen in Figure 4, where contours of the temperature perturbation, with respect to the time average, are presented. A single perturbation structure, marked in blue, is seen to travel up the heated wall, transit below the upper horizontal boundary, and progress into the cold wall boundary layer, demonstrating that the waves are circulating around the cavity in the flow direction. The waves result from a convective instability of the boundary layer whereby the boundary layer above a critical location on the heated wall, and below a critical location on the cooled wall, is unstable to travelling waves. The complexity of this flow, combining vertical natural convection and horizontal intrusion boundary layers, stagnation corner flows, a stratified 

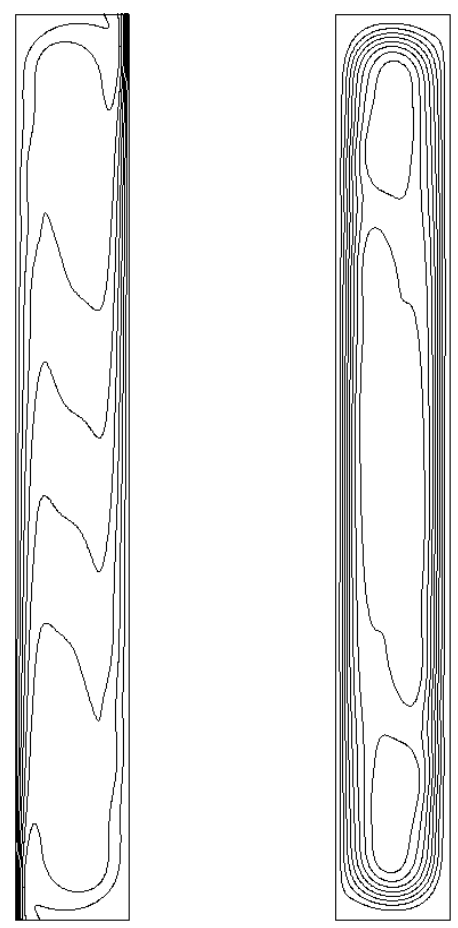

Figure 2: Temperature (left) and streamfunction (right) contours.

core, stable and unstable regions and travelling waves, make it an excellent benchmark for assessing the relative performance of unsteady Navier-Stokes solvers. Full details of the performance of the twenty two solvers considered at the conference, for a range of metrics, are given in [2].

Non-staggered grids store all the unknowns at the same locations, as shown in Figure 5 where both non-staggered and staggered grid configurations are shown. In the past many non-staggered grid Navier-Stokes solvers have been found to suffer from grid-scale oscillations in the pressure field that adversely affect their performance [3]. A number of approaches have been used to prevent the pressure oscillations, all of which effectively 


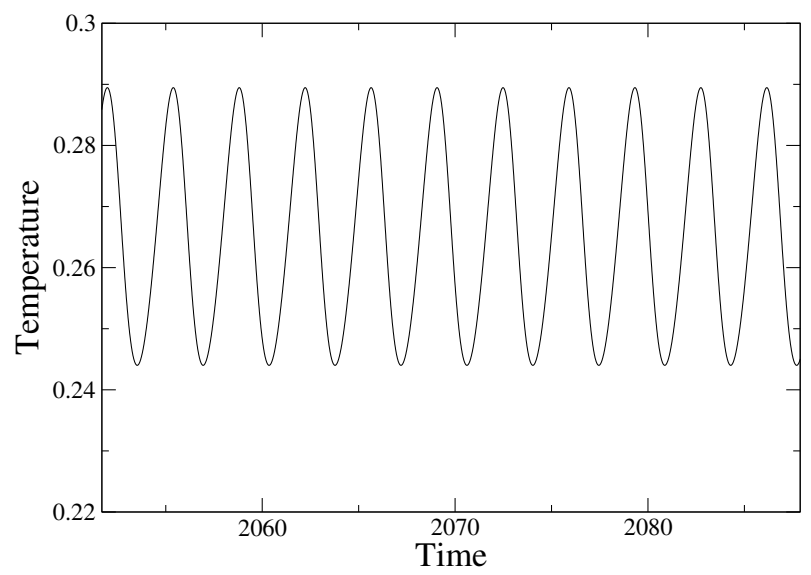

Figure 3: Temperature time series at $x=0.1810, y=7.3700(\bullet$ in Figure 1).

modify the continuity equation by the inclusion of a biharmonic pressure term $[3,4,5,6,7]$. Such an approach removes the problem of pressure oscillations at the expense of the additional error associated with the inclusion of the biharmonic operator. These schemes were all derived in the context of iterative solvers.

Staggered grid schemes automatically couple the grid scale pressure to the remainder of the solution, and thus do not suffer from the problem of pressure oscillation [3]. Figure 5 shows the layout of the standard staggered grid, where the velocities are stored at locations offset from the pressure storage locations in their respective directions. The staggered scheme does have a number of disadvantages when compared to the non-staggered scheme. Each of the velocity components and the pressure have separate control volumes, leading to more complex coding, and additional interpolations are needed to obtain quantities on control volume boundaries.

In contrast to the iterative non-staggered grid solvers, it has been found that non-iterative fractional-step Navier-Stokes solvers, that is schemes in 


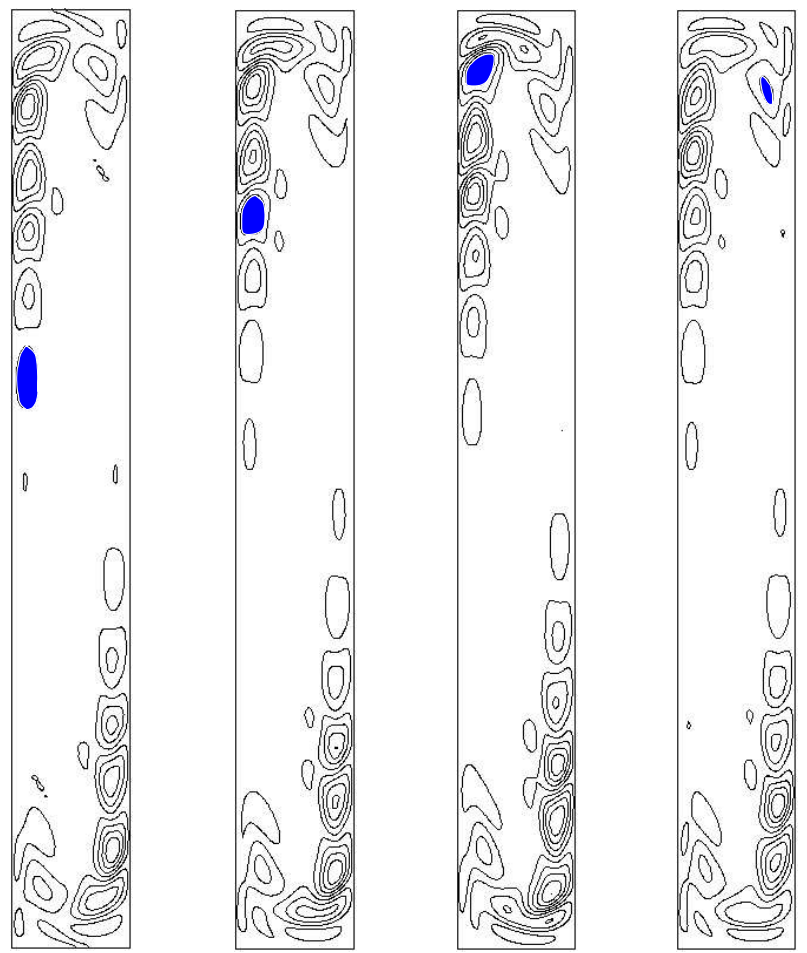

FigurE 4: Temperature perturbation contours with time increasing left to right.
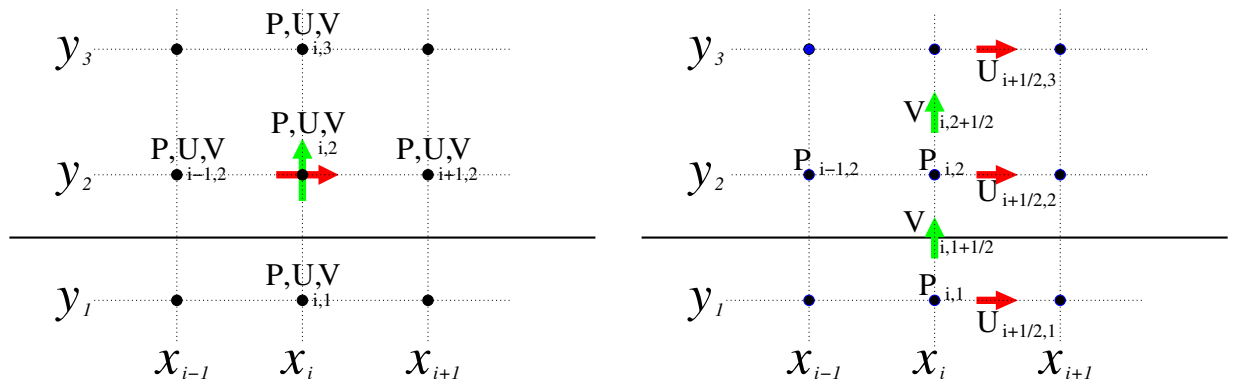

FiguRE 5: Non-staggered grid (left), staggered grid (right) 
which the momentum/pressure equations system is only solved once at each time step, do not require the explicit modification of the continuity equation to ensure good performance and to prevent pressure oscillations. The nonstaggered fractional-step Navier-Stokes solver has previously been shown to be second-order in time [8]. Here the computational efficiency and spatial accuracy of the non-staggered grid scheme is compared directly to that of an equivalent staggered grid scheme for the bifurcated 8:1 cavity natural convection flow.

\section{Method}

The method and its accuracy are presented primarily for the non-staggered grid scheme. The staggered grid scheme is similar and the differences are noted below. The governing equations are the two-dimensional Navier-Stokes equations with the Oberbeck-Boussinesq approximation for buoyancy, together with a temperature transport equation,

$$
\begin{aligned}
u_{t}+(u \cdot \nabla) u & =-\nabla P+\frac{1}{\operatorname{Re}} \nabla^{2} u+j \frac{\operatorname{Ra}}{\operatorname{Re}^{2} \operatorname{Pr}} \tau, \\
\nabla \cdot u & =0, \\
\tau_{t}+(u \cdot \nabla) \tau & =\frac{1}{\operatorname{Pr} \operatorname{Re}} \nabla^{2} \tau,
\end{aligned}
$$

where $u$ is the velocity, $P$ the pressure, $\tau$ the temperature and Re the Reynolds number. The gravity acts in the negative $y$ direction, $j=1$ in the $y$ momentum equation and $j=0$ in the $x$ momentum equation. The Reynolds number $\operatorname{Re}=\bar{U} W / \nu$ with $\bar{U}=\sqrt{g \beta W \Delta T}$, and may be written terms of the Rayleigh and Prandtl numbers as $\mathrm{Re}=\sqrt{\mathrm{Ra} / \mathrm{Pr}}$.

The continuous equations are discretised in time using Adams-Bashforth for the advective terms and Crank-Nicolson for the diffusive terms; for the 
momentum and continuity equations this gives the system $[9,10]$

$$
\begin{aligned}
\frac{v^{n+1}-v^{n}}{\Delta t}+\left[\frac{3}{2} A\left(v^{n}\right)-\frac{1}{2} A\left(v^{n-1}\right)\right]= & -G p^{n+1 / 2}+\frac{1}{2 \operatorname{Re}} L\left(v^{n+1}+v^{n}\right) \\
& +j \frac{\operatorname{Ra}}{\operatorname{Re}^{2} \operatorname{Pr}} T^{n+1 / 2}, \\
D v^{n+1}= & ,
\end{aligned}
$$

where $(v, p, T)$ are the discrete velocity, pressure and temperature respectively, $A$ is the discrete advection operator, $G$ the discrete gradient, $L$ the discrete Laplace operator and $D$ the discrete divergence. Second-order centraldifference discretisations are used for the operators $A, G$ and $D$. Equation (4) is a second order in time representation of equation (1) at the $n+1 / 2$ time location. The temperature equation is discretised in the same manner as the momentum equations, and solved before the momentum equations at each time step, allowing the buoyancy term in the vertical momentum equation to be constructed from $T^{n+1}$ and $T^{n}$. For brevity the buoyancy term will be dropped from equation (4) and only the momentum and associated pressure correction equation considered in the description below.

Fractional-step methods integrate equations (4) and (5) in a segregated manner, that is the momentum equations are first solved for the velocity, and some form of Poisson equation is then solved for the pressure. The Poisson equation is constructed from the momentum equation and the continuity equation and, as well as providing the pressure, acts to enforce continuity.

In this method equation (4) is solved, using the best current value for $p$, to obtain $v^{*}$, an approximation to $v^{n+1}$, that is

$$
\frac{v^{*}-v^{n}}{\Delta t}+\left[\frac{3}{2} A\left(v^{n}\right)-\frac{1}{2} A\left(v^{n-1}\right)\right]=-G p^{n-1 / 2}+\frac{1}{2 \operatorname{Re}} L\left(v^{*}+v^{n}\right),
$$

where the $n-1 / 2$ time-level pressure is that obtained at the previous timestep. This approximate velocity will not initially satisfy continuity. A correction is then applied of the form,

$$
v^{n+1}=v^{*}-\Delta t G \pi,
$$


where $\pi$ is a pressure correction, such that the resulting $v^{n+1}$ does satisfy continuity. An equation for $\pi$ is constructed by substituting equation (7) into the continuity equation (5), to give

$$
L \pi=D v^{*} / \Delta t,
$$

where $L=D G$.

Once $\pi$ is obtained, the velocity is corrected and the pressure is updated using the pressure correction as

$$
p^{n+1 / 2}=p^{n-1 / 2}+\pi .
$$

The $v^{*}$ field is then corrected as above and the integration continues to the next time-step.

The time integration is identical and as described above for both the staggered and non-staggered grid schemes.

\subsection{Accuracy}

\subsubsection{Divergence error}

On the non-staggered grid the discrete Poisson equation for $\pi$ is

$$
\begin{aligned}
&\left(\frac{\pi^{i+1, j}-2 \pi^{i, j}+\pi^{i-1, j}}{\Delta x^{2}}\right)+\left(\frac{\pi^{i, j+1}-2 \pi^{i, j}+\pi^{i, j-1}}{\Delta y^{2}}\right)= \\
& \frac{1}{\Delta t}\left(\frac{U^{i+1 / 2, j}-U^{i-1 / 2, j}}{\Delta x}+\frac{V^{i, j+1 / 2}-V^{i, j-1 / 2}}{\Delta y}\right)^{*} .
\end{aligned}
$$

This Poisson equation has been constructed by discretising the continuous form of equation (8) using centred second-order discretisations. On the nonstaggered grid the.$^{i \pm 1 / 2}$ and.${ }^{j \pm 1 / 2}$ values are obtained by linear interpolation 
from the nodal values. On the staggered grid these values are available and no interpolation is required.

The use of the compact discretisation, given in equation (10), introduces an error into continuity, which may be seen by substituting the corrected form of the velocity into the continuity equation, giving

$$
\begin{aligned}
& \left(\frac{U^{i+1 / 2, j}-U^{i-1 / 2, j}}{\Delta x}+\frac{V^{i, j+1 / 2}-V^{i, j-1 / 2}}{\Delta y}\right)^{n+1}= \\
& \Delta t\left[\left(\frac{\pi^{i+1, j}-2 \pi^{i, j}+\pi^{i-1, j}}{\Delta x^{2}}\right)+\left(\frac{\pi^{i, j+1}-2 \pi^{i, j}+\pi^{i, j-1}}{\Delta y^{2}}\right)-\right. \\
& \left.\left(\frac{\pi^{i+2, j}-2 \pi^{i, j}+\pi^{i-2, j}}{4 \Delta x^{2}}\right)+\left(\frac{\pi^{i, j+2}-2 \pi^{i, j}+\pi^{i, j-2}}{4 \Delta y^{2}}\right)\right] .
\end{aligned}
$$

The right hand side error term is the difference between compact and sparse discretisations of the Laplace operator. The sparse discretisation has a computational molecule with $2 \Delta x, 2 \Delta y$ spacing between the nodes, whereas the compact discretisation has $\Delta x, \Delta y$ spacing, as seen here. This structure results from the use of a compact Laplace operator in equation (10), and the error may be written to second order as

$$
-\Delta t\left[\Delta x^{2} \pi_{x x x x}+\Delta y^{2} \pi_{y y y y}\right] .
$$

Thus, using the compact Laplacian leads to an error in continuity proportional to the fourth spatial derivative of the pressure correction $\pi$. Noting that $\pi \sim \Delta t p_{t}$ means that this error is second order in time, and may be written as

$$
-\Delta t^{2}\left[\Delta x^{2} p_{t x x x x}+\Delta y^{2} p_{\text {tyyyy }}\right] .
$$

The advantage of the staggered grid scheme, in comparison to the nonstaggered grid, is that when the Laplace operator for $\pi$ is constructed directly from the discrete forms of the divergence and gradient operators, a compact discretisation is obtained which does not produce an error in continuity.

Constructing the discrete form of the Laplace operator directly from the discrete forms of the divergence and gradient operators on the non-staggered 
grid, with centred second-order differences used for $D$ and $G$, gives a sparse discrete Laplace operator of the form,

$$
\left(\frac{\pi^{i+2, j}-2 \pi^{i, j}+\pi^{i-2, j}}{4 \Delta x^{2}}\right)+\left(\frac{\pi^{i, j+2}-2 \pi^{i, j}+\pi^{i, j-2}}{4 \Delta y^{2}}\right) .
$$

The use of this sparse operator approach has been found to be inefficient and to lead directly to the pressure oscillations observed in iterative non-staggered schemes.

\subsubsection{Velocity and pressure error}

The velocity field $v^{n+1}$ satisfies the following approximate form of the momentum equations:

$$
\begin{aligned}
\frac{v^{n+1}-v^{n}}{\Delta t}+\left[\frac{3}{2} A\left(v^{n}\right)\right. & \left.-\frac{1}{2} A\left(v^{n-1}\right)\right]=-G\left(p^{n-1 / 2}+\pi\right) \\
& +\frac{\Delta t}{2 \operatorname{Re}} L G \pi+\frac{1}{2 \operatorname{Re}} L\left(v^{n+1}+v^{n}\right),
\end{aligned}
$$

obtained by substituting $v^{*}=v^{n+1}+\Delta t G \pi$, from equation (7), into equation (4). Given $p^{n+1 / 2}=p^{n-1 / 2}+\pi$ the term $(\Delta t / 2 \operatorname{Re}) L G \pi$ is seen to be an error term. This term, which cannot be written as the gradient of a scalar and absorbed into the pressure because the $L$ and $G$ operators do not commute at the boundary, contributes a second-order in time error, as $\pi \sim \Delta t p_{t}$. This error term exists for both staggered and non-staggered grid schemes, and the velocity is second-order accurate in time for both schemes $[8,9,10,11]$.

No explicit equation is solved for the pressure; however, it will satisfy the equation obtained by taking the divergence of equation (12); on the nonstaggered grid this gives

$$
\frac{\left(p^{i+2, j}-2 p^{i, j}+p^{i-2, j}\right)^{n+1 / 2}}{4 \Delta x^{2}}+\frac{\left(p^{i, j+2}-2 p^{i, j}+p^{i, j-2}\right)^{n+1 / 2}}{4 \Delta y^{2}}
$$




$$
\begin{aligned}
& =-D\left[\frac{3}{2} A\left(v^{n}\right)-\frac{1}{2} A\left(v^{n-1}\right)-\frac{\Delta t}{2 \operatorname{Re}} L G \pi\right] \\
& +\Delta t^{2}\left[\Delta x^{2} p_{x x x x}+\Delta y^{2} p_{y y y y}\right]_{t t}-\frac{1}{\operatorname{Re}} L \Delta t^{2}\left[\Delta x^{2} p_{x x x x}+\Delta y^{2} p_{y y y y}\right]_{t} .
\end{aligned}
$$

The use of centred difference schemes for the divergence and gradient operators on a non-staggered grid produces the sparse Laplace operator for pressure shown here. The $(\Delta t / 2 \operatorname{Re}) L G \pi$ term on the second line is a second order in time error term resulting from the use of the fractional-step method, whereas the terms on the third line are a result of the error shown in equation (11). When an iterative scheme is used the momentum/Poisson $\pi$ equations system is iterated until $\pi$ becomes small, the relation $p_{t}=\pi$ no longer holds and these terms are negligible. The fourth derivative of pressure error terms act to limit the growth of grid-scale error in the pressure field, ensuring that the non-iterative scheme is well behaved when the identical scheme, solved in iterative form, behaves poorly. These error terms are all second-order and the pressure is therefore second-order accurate in time at time location $n+1 / 2$. Note that some care must be taken to demonstrate this second-order behaviour, taking into account the $\Delta t / 2$ difference in the location of the pressure and velocities [11].

The implied pressure equation for the staggered grid scheme, equivalent to equation (14), has a compact Laplace operator for pressure and does not include the error terms on the third line of (14).

\subsubsection{Boundary condition error}

Both the $U$ and $V$ velocity components are fixed at a solid wall and the average of the values at the immediate interior and exterior nodes is set to the required value. Because the normal component of velocity is known at the boundary no correction is required to the ${ }^{*}$ field there, and therefore the normal gradient of $\pi$ is set to zero at the boundary. The boundary conditions for the ${ }^{*}$ velocity field are set to be the same as the physical 
boundary conditions, given above. For the non-staggered scheme the normal momentum equation at the immediate interior node requires the pressure at the immediate exterior node. This value is obtained using a fifth order extrapolation from the interior. The boundary extrapolation required for the pressure on the non-staggered grid introduces an additional error component to the order of the extrapolation. The error leads to an error in the velocity of order $\Delta t$ times the order of the extrapolation times the grid-scale squared. For high order extrapolation this error is likely to be negligible.

\section{Results and discussion}

Results have been obtained for $\mathrm{Ra}=3.4 \times 10^{5}$ and $\operatorname{Pr}=0.71$ on two grids. Details of the grids, stretching and time-step are given in Table 1. For each of the grids the smallest mesh is located adjacent to the solid boundaries, and the mesh is stretched away from the boundary with the stretching rate given. The stretching factor is then progressively reduced giving a uniform grid in the interior of the domain. The flow is initialised with a zero velocity and temperature. At time $t=0$ the left and right walls are impulsively heated and cooled to temperatures of 0.5 and -0.5 respectively and the flow is allowed to develop. By time $t=500$ the start up transients have decayed and the timeseries show an oscillatory signal. The momentum and temperature equations are solved using an ADI scheme while the Poisson $\pi$ equation is solved using a restarted GMRES method. The momentum and temperature equations are converged when the absolute residual of the equations, integrated over the domain, is less than $1 \times 10^{-8}$. The $\pi$ equation is converged when the absolute residual, integrated over the domain, is less than $1 \times 10^{-4}$.

The results obtained with the staggered and non-staggered grid schemes and the benchmark results, from [2], are shown in Table 2 in the form of the average, period and amplitude of the temperature time series at location $x=0.181, y=7.37$ (shown as the $\bullet$ on Figure 1) for the fully developed 
TABLE 1: Grid details

\begin{tabular}{|llccc|}
\hline Grid & & $\Delta x, \Delta y$ at wall & Stretching & $\Delta t$ \\
\hline G1 & $451 \times 653$ & $0.000625,0.00125$ & 1.0175 & 0.005 \\
G2 & $211 \times 311$ & $0.00125,0.0025$ & 1.035 & 0.01 \\
\hline
\end{tabular}

TABLE 2: Time series results for temperature at $x=0.181, y=7.37$

\begin{tabular}{|l|ccc|}
\hline & Average & Period & Amplitude \\
\hline Spectral [2] & 0.265480 & 3.41150 & 0.042740 \\
Staggered G1 & 0.265485 & 3.41402 & 0.043195 \\
Staggered G2 & 0.265538 & 3.42347 & 0.043918 \\
Non-Staggered G1 & 0.265490 & 3.41326 & 0.043475 \\
Non-Staggered G2 & 0.265552 & 3.41869 & 0.045448 \\
\hline
\end{tabular}

flow. The errors for the staggered and non-staggered scheme results, with respect to the benchmark results, are shown in Table 3. In absolute terms the difference in accuracy between the staggered and non-staggered schemes is very small. Comparing the results on the $G 2$ grid, the non-staggered scheme is more accurate for the period, while the staggered scheme is more accurate for the amplitude, suggesting that the staggered scheme has a larger dispersive error and the non-staggered scheme has a larger diffusive error. Both schemes give very similar results for the average.

The non-staggered scheme displays second-order convergence for the period and the amplitude, whereas the staggered scheme displays second-order

TABLE 3: Error results for temperature, $x=0.181, y=7.37$

\begin{tabular}{|l|ccc|}
\hline & Average & Period & Amplitude \\
\hline Staggered G1 & $5 \times 10^{-6}$ & 0.00252 & 0.000455 \\
Staggered G2 & $5.8 \times 10^{-5}$ & 0.01197 & 0.001178 \\
Non-Staggered G1 & $1 \times 10^{-5}$ & 0.00176 & 0.000735 \\
Non-Staggered G2 & $7.2 \times 10^{-5}$ & 0.00719 & 0.002718 \\
\hline
\end{tabular}


convergence for the period only. The errors in the average, particularly on the $G 1$ grid, are very small and close to the accuracy with which the benchmark result is presented, and this may be affecting the observed behaviour for this quantity.

Computation times for the two schemes are very similar. On a Pentium 4 with 500 Mbytes of PC2800 DDR memory, execution time for the staggered scheme on grid $G 2$ is 0.805 seconds/time-step, while for the non-staggered scheme it is, 0.836 seconds/time-step. On the $G 1$ grid execution time for the staggered scheme is 3.54 seconds/time-step and for the non-staggered scheme it is 3.44 seconds/time-step.

\section{Conclusions}

In summary, the accuracy and efficiency of the staggered and non-staggered grid schemes are very similar. In particular, note that the good performance of the non-staggered grid scheme is obtained without the inclusion of explicit correction terms into the continuity equation, as is required for iterative schemes. The error that automatically results from using a non-iterative fractional-step method performs the same function as the correction terms do in the iterative scheme, effectively coupling the grid-scale pressure into the remainder of the solution and ensuring the non-staggered scheme has approximately the same performance as the staggered scheme.

Acknowledgment We acknowledge the support of the UPS visiting professor scheme in Civil and Environmental Engineering at Stanford University and the Physical Meteorology Program of the National Science Foundation. 


\section{References}

[1] K. J. Bathe (ed). First MIT Meeting on Computational Solid and Fluid Mechanics, vols 1 and 2, MIT Elsevier, New York, June 2001. C919

[2] M. A. Christon, P. M. Gresho and S. B. Sutton. Computational predictability of time-dependent natural convection flows in enclosures (incuding a benchmark solution). International Journal for Numerical Methods in Fluids, 40:951-1144, 2002.

http://www3.interscience.wiley.com/cgi-bin/jissue/99019470 C921, C930, C931

[3] S. W. Armfield. Finite difference solutions of the Navier-Stokes equations on staggered and non-staggered grids. Computers and Fluids,20:1-17, 1991. http:

//www3.interscience.wiley.com/cgi-bin/abstract/89011177 C921, C922

[4] J. C. Strikewerda. Finite difference methods for the Stokes and Navier-Stokes equations. SIAM J. Sci. Stat. Comput., 5:56-68, 1984. C922

[5] S. Abdallah. Numerical solutions of the pressure Poisson equation with Neumann boundary conditions using a non-staggered grid, 1. J. Computational Physics, 70:182-192, 1987. C922

[6] G. Schneider and M. Raw. Control volume finite-element method for heat transfer and fluid flow using collocated variables-1. Computational procedure. Numerical Heat Transfer, 11:363-390, 1987. C922

[7] C. M. Rhie and W. L. Chow. A numerical study of the turbulent flow past an airfoil with trailing edge separation. AIAA-82-0988, 1982. C922 
[8] S. W. Armfield and R. Street. Fractional step methods for the Navier-Stokes equations on non-staggered grids. ANZIAM J., 42(E):C134-C156, 2000.

http://anziamj. austms.org.au/V42/CTAC99/Armf. C924, C928

[9] S. W. Armfield and R. Street. An analysis and comparison of the time accuracy of fractional step methods for the Navier-Stokes equations on staggered grids. Int. J. Numerical Methods in Fluids, 38:255-282, 2002. C925, C928

[10] S. W. Armfield and R. Street. The fractional step method for the Navier-Stokes equations on staggered grids: the accuracy of three variations. Journal of Computational Physics, 153:660-665, 1999. C925, C928

[11] S. W. Armfield and R. Street. The pressure accuracy of fractional-step methods for the Navier-Stokes equations on staggered grids. ANZIAM J. , 44(E):C20-C39, 2003. http://anziamj.austms.org.au/V44/CTAC2001/Armf C928, C929 